\title{
Couplage entre essais et simulation en soudage
}

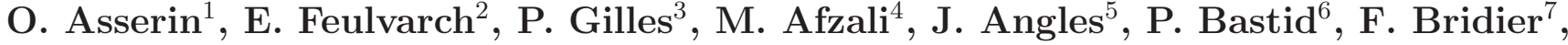

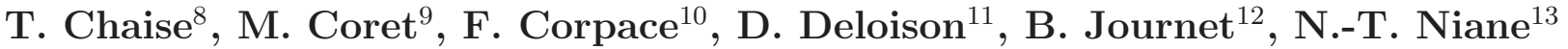 \\ et F. Rossillon ${ }^{14}$
}

Il existe de nombreux essais de soudage : essais de qualification des soudeurs et de mode opératoire, essais de caractérisation des soudures, essais d'évaluation de la performance des assemblages. Depuis la montée en puissance de la simulation numérique, se sont imposés les essais de mise au point des modèles et de leur validation. La maturité aujourd'hui acquise de la modélisation des effets du soudage sur la pièce permet le recours à la simulation numérique pour concevoir des essais de soudabilité et pour comprendre le rôle des contraintes résiduelles dans les essais d'intégrité des structures soudées. Par ailleurs, la simulation des procédés commence à servir à leur amélioration. On peut citer à titre d'exemples : la maitrise de la pénétration latérale, la définition de la forme des buses de gaz de protection ou la compréhension des phénomènes d'instabilité du plasma. C'est dans le domaine de la simulation des essais de fissuration que les modèles font le plus souvent défaut, trop frustres encore pour permettre la simulation des phénomènes.

De façon classique, se pose la double question : quels essais pour la simulation et quelles simulations pour les essais? Mais il est aussi souhaitable de faire émerger les approches où le couplage essais-simulation peut faire progresser l'interaction des équipes chargées d'essais et de calcul dans la recherche de solutions industrielles pour la maitrise des procédés de soudage et la performance des assemblages soudés.

L'approche traditionnelle consiste souvent à faire une dichotomie entre les essais et la simulation, il n'est pas rare d'ailleurs que les deux mondes aient du mal à communiquer. On considère ainsi souvent les essais comme source pour alimenter des modèles de simulation par exemple pour la caractérisation des propriétés des matériaux, la validation des modèles par corrélation essais - calcul, ou bien la simulation comme outil d'aide à l'interprétation des essais pour l'identification et la compréhension de phénomènes qui dépassent l'homme de l'art. Pour faire une analogie numérique on parlerait de couplage faible essais-simulation.

Les papiers présentés sont à dominante industrielle et montrent que des efforts sont fait pour dépasser ce point de vue avec une bonne synergie entre le monde académique et industriel. Notamment avec :

\footnotetext{
${ }^{1}$ Membre du comité éditorial de Matériaux et Techniques, CEA, DEN, DANS, DM2S, 91191 Gif-sur-Yvette, France

2 ENISE Univ Lyon, 58 rue Jean Parot, Saint-Étienne Cedex 2, France

3 AREVA-NP, Paris La Défensse Cedex, France

4 CETIM, Senlis, 52 av. Félix-Louat, BP 67, Senlis, France

5 EDF/R\&D/MRI, France

6 TWI Ltd, Granta Park, Great Abington, CB1 6AL, Cambridge, UK

7 DCNS Research, Indret, 44620 La Montagne, France

LaMCoS, INSA-Lyon, 18-20 rue des Sciences, 69621 Villeurbanne Cedex, France

9 École centrale de Nantes, 1 rue de la Noë, BP 92101, 44321 Nantes Cedex 3, France

10 SNECMA, Groupe SAFRAN, SNECMA site d'Evry-Corbeil, Rue Henri-Auguste Desbruères, BP-81, 91003 Evry Cedex, France

11 EADS Innovation Works, 12 rue Pasteur, BP 76, 92152 Suresnes Cedex, France

12 EADS CCR, 12 rue Pasteur, BP 76, 92152 Suresnes Cedex, France

13 PSA Peugeot Citroën, 18 rue des Fauvelles, 92250 La Garenne Colombes, France

14 EDF SEPTEN, Villeurbanne, France
} 
- les apports de la simulation dans l'interprétation d'essais par exemple pour la fissuration à chaud ou à froid,

- la simulation des essais en soudage (essais de mise au point, de caractérisation, d'endurance, de qualification, de laboratoire) dans le cas de pièces à grands nombres de composants ou nécessitant des assemblages hétérogènes.

- des essais de caractérisations des propriétés des matériaux pour les modèles

- des éléments de compréhension des limites d'un modèle afin de l'améliorer.

De plus, s'il y a quelques années la simulation numérique du soudage se résumait à une simulation thermomécanique qui ne regardait que les effets induits du procédé sur la pièce comme les contraintes résiduelles et les déformations, force est de constater que ce n'est plus le cas aujourd'hui avec l'apparition de plus en plus fréquentes de simulations multiphysiques qui traitent des effets du procédé sur la soudabilité. Une étape a été clairement franchie mais un lien plus fort avec le monde expérimental sera nécessaire pour mesurer les quantités physiques pertinentes pour la validation de ces nouveaux modèles intégrant plus de physiques, avec en particulier le développement de nouvelles méthodes de mesures idoines.

On pourra ensuite espérer augmenter la performance des essais en « exacerbant » les phénomènes à observer, en définissant par simulation les observables pertinentes, leurs plages de variations, leurs positions, l'instrumentation à mettre en place. Bref, utiliser la simulation numérique pour concevoir des essais d'interprétation plus pertinents qui à leur tour serviront à évaluer les modèles utilisés dans la simulation.

Dans ce numéro spécial, les articles présentés sont des contributions au $12^{\mathrm{e}}$ séminaire du Groupement Scientifique et Technique «Simulation Numérique du Soudage (SNS) de l'AFM (Association Français de la Mécanique), organisé autour de la thématique du couplage essais-simulation pour la conception et interprétation des essais en soudage.

Ces publications devraient vous aider à mieux concevoir le soudage grâce aux deux yeux de l'expérimentation et de la simulation. Le troisième oil restant bien entendu celui de l'expert. 